\title{
Analysis of Breast Parenchymal Density on Mammograms in relation to Age and Parity among northeastern Nigerian women
}

\author{
L. L. Hamman ${ }^{1}$, D.S.Amaza ${ }^{1}$, Z. Mustapha ${ }^{2}$, J. V. Zirahei ${ }^{1}$, A. K. Muhsin ${ }^{1} \&$ H. \\ Japhet \\ ${ }^{l}$ Department of Human Anatomy, College of Medical Sciences, University of Maiduguri, Borno State, Nigeria. \\ ${ }^{2}$ Department of Radiology, University of Maiduguri Teaching Hospital(UMTH), Maiduguri, Borno State,
} Nigeria.

\begin{abstract}
AIMS: To Correlate Breast Parenchymal Densities among Northern Women who attended Clinic Screening in relation to age and Parity.

Study design: Retrospective study.

Place and Duration of Study: Department of Human Anatomy College of Medical Sciences University of Maiduguri and Department of Radiology, University of Maiduguri Teaching Hospital (UMTH), between July 2010 and March 2011.

Methodology: Parenchymal densities of Screened mammograms were assessed visually on both cranio-caudal and medio-lateral oblique views. The documented parenchymal densities were categorised using BIRADS categorization based on the 'BR' technique used in Department of Radiology, University of Maiduguri Teaching Hospital (UMTH). The 'BIRADS' technique involves four values given to a mammogram depending on its density.

BR1 - Predominantly Fatty; BR2 - Scattered Fibro glandular Densities

BR3 - Heterogenously Dense; BR4 - Very Dense Breast Tissue

The subcutaneous and retromammary fat regions in each breast were excluded during the determination of dense tissue. In patients with asymmetric tissue, breast with greater density was categorized. For each patient, benign or probably benign calcifications were not recorded.

Results: Parenchymal density on mammograms decreased progressively from the age cohort of 25-29 years old in which $38 \%$ had predominantly fatty breasts through the cohort of 75-79 years old, in which 100\% had predominantly fatty breasts. In cohort of 40-44 years, 32\% had scattered fibro glandular densities. In cohort of 45-49 years, $31 \%$ of patient had scattered parenchymal densities. Five or more pregnancies were associated with predominantly fatty breasts in 34 women compared with null parity or one pregnancy.

Conclusion: Younger age and fewer than two pregnancies are significantly associated with, but not highly predictive of increased parenchymal density on mammograms. Significant percentages (38\%) of women 25-29 years old have predominantly fatty breast tissue on mammograms that should not impede selective mammographic screening or diagnostic effort in this age group. Further investigations could be carried out with other parameters such as benign or malignant calcifications seen on mammographs so as to predict and prevent breast cancer at early stage.
\end{abstract}

Keywords: [Breast, parenchymal density, mammogram, northern Nigeria]

\section{Introduction} that of fat [1]

The term mammographic density signifies areas within the breast seen radiographically over and above

The mammas (breast) secrete the milk and are accessory glands of the reproductive system. They exist in the male as well as in the female; but in the former only in the rudimentary state, unless their growth is excited by peculiar circumstances. In the female they are two hemispherical eminences (variable in size) lying within the superficial fascia and situated on the front and sides of the chest; each extends from the second rib above to the sixth rib below, and from the side of the sternum to near the mid-axillary line.

Their weight and dimensions differ at different periods of life, and in different individuals. Before puberty they are of small size, but enlarge as the generative organs become more completely developed. They increase during pregnancy and especially after delivery, and become atrophied in old age [2]

Mammography is the process of using low-dose amplitude-X-rays (usually around $0.7 \mathrm{mSv}$ ) to examine the human breast and is used as a diagnostic and a screening tool. A Mammography exam, called a mammogram, is used to aid in the early detection and diagnosis of breast diseases in women. An x-ray (radiograph) is a noninvasive medical test that helps physicians diagnose and treat medical conditions. Imaging 
Analysis of Breast Parenchymal Density on Mammograms in relation to Age and Parity among with $\mathrm{x}$-rays involves exposing a part of the body to a small dose of ionizing radiation to produce pictures of the inside of the body. X-rays are the oldest and most frequently used form of medical imaging [1]

Radiographically the breast consists mainly of two component tissues: fibro-glandular tissue and fat. Fibroglandular tissue is a mixture of fibrous connective tissue (the stroma) and the functional (or glandular) epithelial cells that line the ducts of the breast (the parenchyma) [3].

Fat has a lower X-ray attenuation coefficient than fibro-glandular tissue and, therefore, is more transparent to X-rays. Thus, regions of fat appear darker on a radiograph of the breast. Regions of brightness associated with fibro-glandular tissue are referred to as 'mammographic density'. From the pattern of brightness in a mammographic image, the relative prevalence of these tissues in the breast can be inferred [3].

\section{Material And Methods}

The mammography equipment is composed of these main components; Dedicated mammography stand, Exposure control console, Image recording device (cassette or digital), Mammography generator. A mammography viewing box, UMTH mammography data sheets, tables and UMTH mammograms were used to collect and document relevant data. All women in the study had their mammograms done with a GE Senographe DMR mammography unit and, their mammograms where taken on mammography films, Agfa brand, size $18 \mathrm{~cm}$ $\mathrm{x} 24 \mathrm{~cm}$ and $24 \mathrm{~cm} \times 30 \mathrm{~cm}$.

During mammography, breast is positioned in the mammography unit (Fig 1). The breast will be placed on a special platform and compressed with a paddle (often made of clear Plexiglas or other plastic), and gradually compress the breast [4]

\subsection{Research Design}

Parenchymal densities of screening mammograms from women seen in the breast unit of Radiology Department, University of Maiduguri Teaching Hospital (UMTH) were assessed visually on both cranio-caudal and medio-lateral oblique views. The documented parenchymal densities were categorized using BIRADS categorization, and were categorized based on the 'BR' technique used in UMTH.

The 'BIRADS' technique involves four (4) values called BIRADS values given to a mammogram depending on its density.

BR1 - Predominantly Fatty

BR2 - Scattered Fibro glandular Densities

BR3 - Heterogenously Dense

BR4 - Very Dense Breast Tissue

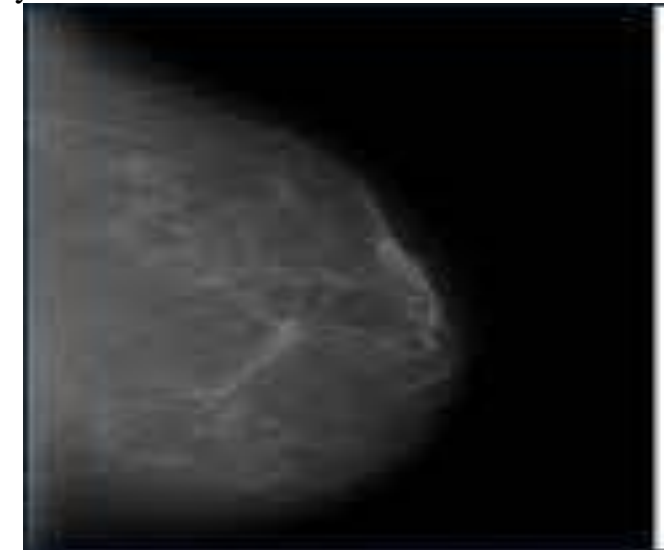

BR1 - Predominantly Fatty

(Found in women 40yrs \& above)

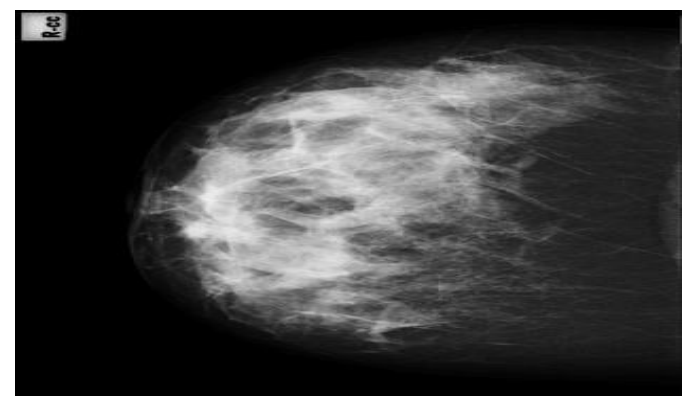

BR3 - Heterogenously Dense

(Found in women 40yrs \& under)

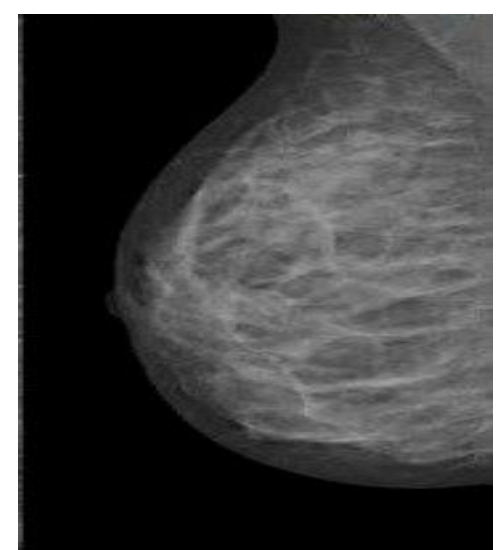

BR2 - Scattered Fibro glandular Densities (Found in women 40yrs \& above)

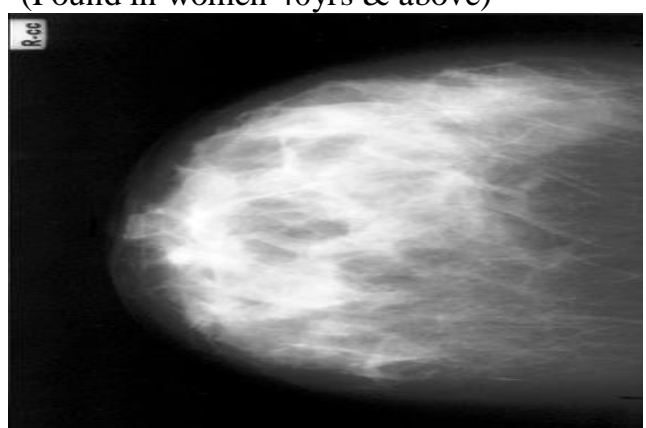

BR4 - Very Dense Breast Tissue

(Found in women 34yrs \& under) 
The subcutaneous and retro-mammary fat regions in each breast were excluded during determination of dense tissue. Patients with asymmetric tissue, the breast with greater density were categorized. Benign or probably benign calcifications were not recorded.

\subsection{Sample Size}

The sample size was analyzed using the stratified sampling formula,

$$
\mathrm{N}=(\mathrm{nh} \times \mathrm{n}) / \mathrm{Nh}
$$

Where $\quad \mathrm{N}=$ Population size (6000)

$$
\begin{aligned}
& \mathrm{n}=\text { Sample size }(\mathrm{n}) \\
& \mathrm{Nh}=\text { Population striation }(2) \\
& \mathrm{nh}=\text { Sample striation }(40)
\end{aligned}
$$

Therefore, $\mathrm{n}=(\mathrm{N} \times \mathrm{Nh}) / \mathrm{nh}=(6000 \times 2) / 40=300$

All values where approximated to the nearest hundreds.

The study population consisted of 300 women grouped into 5-year age cohorts that ranged from 25-29, 30-34, 35-39, 40-44, 45-49, 50-54, 55-59, 60-64, 65-69, 70-74 and 75-79 years old.

\section{Results And Discussion}

The parenchymal density seen on mammograms of women from 25 - 79 old is shown in table 1 .

Table 1. Effects of Parity on Parenchymal Density on Mammograms in Women.

\begin{tabular}{lcccccc}
\hline Factor & $\begin{array}{c}\text { Total no } \\
\text { of Patients }\end{array}$ & $\begin{array}{c}\text { \% age of } \\
\text { patients }\end{array}$ & & \multicolumn{3}{c}{ Breast Densities } \\
\cline { 5 - 7 } & & & & & & \\
\hline Parity & 39 & $13.00 \%$ & NIL & 13 & 12 & 14 \\
Nullparity & 13 & $04.33 \%$ & 6 & NIL & 7 & NIL \\
One Pregnancy & 118 & $39.33 \%$ & 32 & 34 & 33 & 19 \\
Two-five Pregnancies & 130 & $43.33 \%$ & 34 & 58 & 38 & NIL \\
Five above & $\mathbf{3 0 0}$ & $\mathbf{1 0 0}$ & & & & \\
\hline Total & &
\end{tabular}

The parenchymal density on mammograms decreased progressively from the age cohort of 25-29 years old in which $38 \%$ had predominantly fatty breasts through the cohort of 75-79 years old, in which $100 \%$ had

\begin{tabular}{|c|c|c|c|c|c|}
\hline \multirow{2}{*}{$\begin{array}{c}\text { Age } \\
\text { Cohort }\end{array}$} & \multirow{2}{*}{$\begin{array}{c}\text { No of } \\
\text { Individual } \\
(\pi)\end{array}$} & \multicolumn{4}{|c|}{ Parenchymal Densities (No of Patients) } \\
\hline & & $\begin{array}{c}\text { BR1 } \\
(\% \text { age of } \pi)\end{array}$ & $\begin{array}{c}\text { BR2 } \\
(\% \text { age of } \pi)\end{array}$ & $\begin{array}{c}\text { BR3 } \\
(\% \text { age of } \pi)\end{array}$ & $\begin{array}{c}\text { BR4 } \\
(\% \text { age of } \pi)\end{array}$ \\
\hline $25-29$ & 13 & - & - & - & \\
\hline $30-34$ & 27 & $5 \quad(38 \%)$ & $4(31 \%)$ & $4(31 \%)$ & Nil \\
\hline $35-39$ & 35 & $8 \quad(30 \%)$ & $10(37 \%)$ & $7 \quad(26 \%)$ & $2(7 \%)$ \\
\hline $40-44$ & 67 & $8(22.85 \%)$ & $11(31.42 \%)$ & $11(31.42 \%)$ & $5 \quad(14.28 \%)$ \\
\hline $45-49$ & 45 & $17(25.37 \%)$ & $32(46.76 \%)$ & $15(22.38 \%)$ & $3 \quad(4.47 \%)$ \\
\hline $50-54$ & 32 & $10(22.22 \%)$ & $21(46.66 \%)$ & $14(31.11 \%)$ & Nil \\
\hline $55-59$ & 26 & $10(31.25 \%)$ & $13(40.62 \%)$ & $9 \quad(28.12 \%)$ & Nil \\
\hline $60-64$ & 16 & $10(38.46 \%)$ & $8(30.76 \%)$ & $8 \quad(30.76 \%)$ & Nil \\
\hline $65-69$ & 17 & $13(81.25 \%)$ & $3(18.75 \%)$ & Nil & Nil \\
\hline $70-74$ & 12 & $15(88.23 \%)$ & $2(11.76 \%)$ & Nil & Nil \\
\hline \multirow[t]{2}{*}{$75-79$} & 10 & $11(91.66 \%)$ & $1 \quad(8.33 \%)$ & Nil & Nil \\
\hline & & $10(100 \%)$ & Nil & Nil & Nil \\
\hline
\end{tabular}
predominantly fatty breasts. In cohort of 40-44 years, $32 \%$ had scattered fibro glandular densities. In cohort of 45-49 years, $31 \%$ of patient had scattered parenchymal densities.

Table 2. Parenchymal Densities on Mammograms in Women 25 - 79 Years Old.

The relationship between parenchymal densities and parity is shown in table 2. Five or more pregnancies were associated with predominantly fatty breasts in 34 women compared with null parity or one pregnancy (nil and 6 women respectively).

\subsection{DISCUSSION}

Although this study shows that younger age is a factor in parenchymal density seen mammographically, a significant percentage (38\%) of women 25-29 years old (Table 2) have predominantly 
Analysis of Breast Parenchymal Density on Mammograms in relation to Age and Parity among fatty tissue on mammograms. Fewer than two pregnancies (Table 1) is also a significant factor associated with increased breast tissue density in all age groups.

These data may be helpful when considering the use of mammography and new imaging techniques to detect and diagnose breast cancer in younger women.

Physical examination and degree of compressibility have been shown to be inaccurate predictors of parenchymal density on mammograms.

Observation that little difference exists in the proportion of fatty to dense breast tissue in women 40-44, 44-49 and 50-54 years old. This is also in accordance with the work of [5]. This data is noteworthy when considering the age at which mammographic screening begins in the general population.

Data shows no significant change in parenchymal density on mammograms at age 50 that would support initiating screening at age 50 rather than age 40 .

There is an association of decreased parenchymal density on mammograms with increased parity. This is also in line with work of [6].

The knowledge of age-specific frequency of benign calcifications may be important in educating women and health care providers.

Anxious patients often require explanation and reassurance that some mammographic patterns of calcification suggest malignancy and require biopsy whereas benign calcifications are recognized often on mammograms in women of the same age and increase in frequency with age.

An understanding of the biologic factors associated with the development and regression of parenchymal density on mammograms may lead to the development of therapeutic methods that decrease breast density and unmask carcinomas obscured by density tissue on mammograms.

\section{Conclusion}

In conclusion younger age and fewer than two pregnancies are significantly associated with, but not highly predictive of increased parenchymal density on mammograms. Significant percentages (38\%) of women 25-29 years old have predominantly fatty breast tissue on mammograms that should not impede selective mammographic screening or diagnostic effort in this age group.

Further investigations could be carried out with other parameters such as benign or malignant calcifications seen on mammographs so as to predict and prevent breast cancer at an early stage.

\section{Acknowledgements}

The authors are grateful to all the staff of the Mammogram unit of Radiology Department, University of Maiduguri Teaching Hospital (UMTH), for assisting us with the records of the mammographs, and also to the Ethical committee for their kind approval that leads to the success of this study.

\section{Competing Interests}

Authors have declared that no competing interests exist.

\section{References}

[1] Wolfe JN. Breast Parenchymal Patterns and Their Changes with Age. Radiology 121 (3), 121, 1976, 545-52

[2] Robinson GW, Karpf AB, Kratochwil K. Regulation of mammary gland development by tissue interaction. J Mammary Gland Biol Neoplasia; 4(1), 1999, 9-19.

[3] Destounis SV, DiNitto P, Logan-Young W, Bonaccio E, Zuley ML, Willison KM.Can computer-aided detection with double reading of screening mammograms help decrease the false-negative rate? - Initial experience".Radiology 232 (2), 2004, 578-80.

[4] Qaseem A, Snow V, Sherif K, Aronson M, Weiss KB, Owens DK. Screening mammography for women 40 to 49 years of age: a clinical practice guideline from the American College of Physicians. Ann. Intern. Med. 146 (7): 2007, 511-5.

[5] Saftlas AF, Hoover RN, Brinton LA, Szklo M, Olson DR, Salane M, et al. Mammographic Densities And Risk of Breast Cancer.;67, 1991, 2833-2838.

[6] Stomper PC, D'Souza DJ, DiNitto PA, Arredondo MA. Analysis of parenchymal density on mammograms in 1353 women 25-79 years old. Am, J Roentgenol. 167(5), 1996, 1261-5. 